\title{
CHROMOSPHERIC EMISSION LINE MAPPING
}

\author{
JAMES E. NEFF \\ Pennsylvania State University \\ 525 Davey Laboratory \\ University Park, PA 16802
}

\begin{abstract}
.
Throughout its eighteen years of operation, the International Ultraviolet Explorer has afforded us the opportunity to make many new discoveries regarding the nature of the outer atmospheres of magnetically active, latetype stars. High-resolution spectra obtained continuously throughout the rotational cycle of rapidly-rotating stars have been used to map the spatial structure of the magnetic active regions in stellar chromospheres. The RS CVn-type binary system AR Lacertae has been observed intensively at 3 epochs, with additional observations with less complete phase coverage was obtained at 4 other epochs. Taken together, we are able to probe the evolution of atmospheric structure of AR Lac over a 13-year timeline. I briefly review techniques of chromospheric emission line mapping, and I summarize the IUE results for AR Lac, V711 Tau, EI Eri, V1794 Cyg, and TY Pyx. I conclude with a description of chromospheric imaging programs (present and future) that make use of the Hubble Space Telescope.
\end{abstract}

\section{Introduction}

The photospheric imaging problem now appears to be well understood (e.g. Collier Cameron 1992; Unruh \& Collier Cameron 1995), and several groups have presented intriguing results at this meeting. There is a strong temptation to apply the same procedures to ultraviolet spectra in order to produce chromospheric images. It would be premature to expect such detailed maps for several reasons. Until very recently, the existing ultraviolet spectra were of much lower quality than the visible spectra used as input to the photospheric imaging routines. More fundamentally, the basic physics 
of chromospheres is yet to be developed.

To first order, we know the emitting volume of the photosphere, so we can use the observed spectrum to infer its physical properties. We know neither the surface area coverage nor the radial extent of the chromospheric active regions. These are the first properties we must attempt to measure. The actual distribution and shape of the active regions is of secondary interest. Nevertheless, the most straightforward way to measure the size of active regions is to image the chromosphere at several heights, thus synthesizing a three-dimensional picture.

There are several advantages unique to chromospheres or to ultraviolet observations that tend to simplify the problem. The most obvious advantage is contrast: photospheric spots are dark features viewed against a bright background, whereas active regions ("plages") are bright regions viewed against a dark background. Increased activity tends to produce a brighter and broader emission line profile from chromospheres, while it tends to "wash out" photospheric profiles. The contrast advantage implies that the same spatial detail can be inferred from spectra of far lower signalto-noise than required for photospheric studies. This is fortunate because the ultraviolet spectrographs on IUE produce lower-resolution, lower-S/N spectra than ground-based, visible spectrographs. The GHRS on HST produces spectra of quality comparable to those obtained using ground-based spectrographs. Space-based observations are difficult to coordinate and telescope time is very expensive, but there are no sky constraints or day/night cycles in high-earth orbit.

Real stars conspire to defeat some of these advantages. Chromospheres are far more variable than photospheres, so continuous observations are a must. Systematic flows produce line shifts, introducing positional uncertainty. The high-temperature plasma tends to increase the intrinsic line widths, reducing spatial resolution.

\section{Basic principles of spectral imaging}

There are several noteworthy differences between photospheric imaging techniques (e.g., Vogt, Penrod, \& Hatzes 1987; Piskunov, Tuominen, \& Vilhu 1990) and ultraviolet techniques (Walter et al. 1987; Neff et al. 1989). The fundamental difference is that for chromospheres, there is no single parameter that we can map from each grid point on a star to position on a model profile. For the moment, at least, chromospheric spectral imaging must be based on a direct decomposition of emission line profiles.

The basic premise of ultraviolet spectral imaging is that emission arising from a uniform distribution on a stellar surface will produce a symmetric profile centered at the stellar velocity. Non-uniformities of the surface 
brightness distribution will produce emission components that vary in wavelength due to the Doppler shift produced by the star's rotation. The net profile will be asymmetric, and a single-component fit to this profile will not lie at the stellar velocity. Therefore, fitting the observed profiles with a symmetric component centered at the stellar velocity accounts for the uniform part of the spatial distribution. If such a fit matches the observed profiles, then we can say nothing more about the spatial distribution. If, on the other hand, additional components are needed to account for the residual emission or to adjust the velocity centroid of the fit, then there must be a non-uniform component of the spatial distribution.

By applying this very basic concept, we have already separated the spatial distribution into uniform and non-uniform parts. The next step is to repeat this decomposition for spectra obtained at many (at least 10 or 12) different rotational phases. Each phase must be observed at least twice, not for redundancy but simply to produce a spatial map. With repeated phasecoverage, both uniform and non-uniform components can be subdivided into time-dependent (i.e. variable) and time-independent (i.e. constant). The time-dependent parts subdivide one more time into phase-dependent (i.e. periodic) and phase-independent parts. Any feature that has not been seen to repeat at the same phase on at least two orbits cannot be interpreted as phase-dependent. Each of the six components identified in this fashion represent different physical phenomena, not all of them spatial:

1. Uniform and Constant - This component tells us very little, because we cannot say whether emission arises homogenously from the entire surface or from a uniform distribution of active regions.

2. Uniform and Phase-Dependent - Applying only the constraint of symmetry, it possible for the uniform component to vary in intensity and width. If this variation is phase-dependent, then there could be a nonuniformity to the spatial brightness distribution that is too largescale to produce a measurable influence on the line profile (i.e. a hemispheric difference). Binary effects, such as tidal distortion and unforseen eclipses, must also be considered.

3. Uniform and Phase-independent - This could be due to intrinsic variability with an undetermined period or simply stochastic variability taking place on a global scale.

4. Non-uniform and Constant - Assymetries in the line profile that don't vary with time or phase are the most difficult feature to explain. If their position corresponds to the system center of mass, then they could reasonably be interpreted as emission from that region. If they are matched by a similar component on the opposite side of the line profile, then the presumed intrinsic profile should be called into question (e.g. it might not be gaussian). 
5. Non-uniform and Phase-dependent - Asymmetries that transit the line profile from blue to red as the star rotates map into discrete active regions on the stellar surface. Their radial velocity, relative to the mean stellar radial velocity, provides their position. Their line width, if they are resolved, can be used to measure their size. With the size of the region, we can directly measure its surface flux.

6. Non-uniform and Phase-Independent - These features must be due to short-lived phenomena taking place in localized regions. If several spectra are obtained during the lifetime of the event, then the size and location of the region can be measured as if it were phase-dependent.

The mapping of the non-uniform components into discrete regions can be complicated by the dynamics within the various regions. The measured wavelength on the line profile maps directly to a radial velocity, but this radial velocity can be partly rotational and partly dynamical. For example, during flares we see net red-shifts that presumably are caused by a net flow of the flaring plasma (e.g. Linsky et al. 1989). The spatial resolution is proportional to the rotational velocity divided by the intrinsic width. If there are large randomly-directed flows, the intrinsic line width increases, thus decreasing the spatial resolution.

\section{Chromospheric mapping using IUE}

Four short-period RS CVn binary systems and one FK Com system have been observed with the International Ultraviolet Explorer throughout a rotational cycle. In all but a few cases, these observations were obtained over only a single cycle, making it impossible to determine which variations were truly phase dependent. In many cases, flares affected the line profiles for several hours at a time. While intrinsically very interesting, these sporadic events decrease phase coverage available for mapping. In a few cases, we have been able to measure the location, size, surface brightness, and dynamical properties of the flaring plasma using the spectral imaging technique.

\subsection{AR LACERTAE}

AR Lac (=HD 219334) is an eclipsing RS CVn binary system (K0 IV + G2 IV). The two stars rotate synchronously with the orbital period (1.98 days), yielding an equatorial velocity of $72 \mathrm{~km} \mathrm{~s}^{-1}$ for the $\mathrm{K}$ star and 39 $\mathrm{km} \mathrm{s}^{-1}$ for the $\mathrm{G}$ star. Because the system is bright $\left(\mathrm{m}_{\mathrm{v}}=6.1\right)$ and because both stars are active, the system is an ideal candidate for ultraviolet spectral imaging. 
Walter et al. (1987) first developed these ultraviolet spectral imaging techniques to analyze a series of 8 high-dispersion spectra of AR Lac obtained with the IUE satellite in October 1983. The spectra were concentrated near the eclipse phases. This poor phase coverage and resolution and the lack of repeated phase coverage led to ambiguous results. Their work nevertheless demonstrated the potential of the technique and led to a few surprising results. The primary conclusions of Walter et al. (1987) were that (1) both stars are active and globally variable, (2) discrete plage regions are resolvable on the $\mathrm{K}$ star surface, and (3) the far-ultraviolet surface flux spectrum of each component can be obtained from the observed low-resolution spectra.

Armed with confidence in these techniques but requiring bettter phase coverage, we observed AR Lac continuously with IUE for $80 \%$ of a single orbital cycle is September 1985. The final system model for AR Lac in September 1985 included three discrete chromospheric "plage" regions. The $\mathrm{G}$ star was chromospherically inactive between longitudes $335^{\circ}$ and $75^{\circ}$. A flare was observed on the $\mathrm{G}$ star. The flaring region covered at most $2 \%$ of the stellar surface. There was a systematic redshift and turbulent broadening of the flare emission. The global (uniform) flux from both stars varied, as did the plage flux. The primary result from the 1985 observations was not the surface geometry but the surface flux within each region. All of this information was presented as a series of images in Neff et al. (1989).

In September 1987 we observed AR Lac continuously with IUE for two full orbital cycles (4 days). This series provided uniform and complete phase coverage, and observations of the same phase were obtained during subsequent orbits. Both stars were roughly $50 \%$ brighter in their trailing hemisphere than in their leading hemisphere. The overall line widths showed a hemispheric difference in the same sense as the flux. The structure that produces this variation must be very large-scale, or it would produce an asymmetric profile at some phases. There were some noteworthy differences between the two orbits, showing that some of the features were transient. No major flares, however, were observed during this 4 day interval. Preliminary results were presented by Neff (1990) and by Pagano, Rodonò, \& Neff (1992). In December 1991 we observed AR Lac continuously for over two cycles using IUE, HST, ROSAT, the VLA, and various ground-based telescopes. In October 1994 we again arranged for two-cycle coverage of AR Lac, but without the multi-wavelength support.

Only the 1987, 1991, and 1994 observations were obtained over two complete cycles. Incomplete or non-repeated phase coverage of AR Lac was obtained with IUE in 1981 (4 phases), 1983 (8 phases clustered near eclipses), 1985 ( $80 \%$ of a single cycle), 1989 (nearly complete coverage for a single cycle), and 1990 (about half of a single cycle). Taken together, this 
data set permits us to study the evolution of active regions on AR Lac over a 13-year timeline (see Pagano, Rodonò, \& Neff 1994). AR Lac has proven to be an ideal target for ultraviolet spectral imaging. We have used this target to develop and refine the procedures, and it has reminded us that chromospheres are neither static nor homogeneous.

\subsection{V711 TAURI}

V711 Tau (=HR 1099) is the canonical Doppler imaging target system (see the paper by Vogt \& Hatzes in this volume). It was observed frequently with IUE, but only in December 1984, December 1992, and September 1993 were the observations obtained over at least a full cycle (2.8 days). The December 1992 observations were obtained continuously over 6 days and were coordinated with the MUSICOS92 campaign. The most striking results were the frequent and energetic flares (Neff, Pagano, \& Rodonò 1994, 1995). V711 Tau is a relatively slow rotator, and the emission profiles of the two stars are blended. There has been variation of both line wings outside of flares, but it has never showed any clear phase dependence. We are re-analyzing the entire V711 Tau data set in light of the new HST results discussed below (Busa et al. 1995; see also Dempsey et al. in this volume).

\subsection{EI ERIDANI}

EI Eri (=HD 26337) is a single-lined, rapidly-rotating RS CVn system. We observed EI Eri with IUE in September 1988 throughout nearly two complete rotational cycles (Neff 1991). The first rotational cycle was startlingly different from the second cycle. Much of this difference was likely due to flares during the first cycle. The fluxes from the transition region, chromosphere, and photosphere do not seem to correlate well. A paper combining the results based on recently reprocessed IUE data with supporting groundbased visual and radio data is in the final stages of preparation.

\subsection{V1794 CYGNI}

V1794 Cyg (=HD 199178) is another ideal imaging candidate, because it is single (and therefore has no radial velocity variations) and has a $v \sin i$ of $80 \mathrm{~km} \mathrm{~s}^{-1}$. We observed this star with IUE in September 1986 at 6 phases thoughout it's 3.3 day rotational cycle and in September 1987 at 7 phases (Neff 1991). The Mg II emission centroid varied roughly sinusoidally with an amplitude of $\sim 10 \mathrm{~km} \mathrm{~s}^{-1}$, which is well outside the range permitted by ground-based observations and well outside the range of instrumental uncertainties. There must therefore be some non-uniformity to the brightness 
distribution. The $\mathrm{Mg}$ II data can be interpreted as either a time-dependent asymmetry of the red wing or as a large-scale non-uniformity of the chromospheric brightness (or both).

\subsection{TY PYXIDIS}

During the ROSAT all-sky survey, we observed the eclipsing RS CVn binary system TY Pyx (HD 77137) continuously for a single cycle (3.2 days). Supporting observations were obtained from the VLA, various ground-based observatories, and ROSAT. The two G5 IV stars in this system are virtually identical. Their $v \sin i$ is relatively low, but their profiles are unblended. Unfortunately, neither star showed any asymmetry or even uniform variability in their Mg II emission lines, so we were unable to map any chromospheric spatial structure (Neff et al. 1995).

\section{Chromospheric mapping using the Hubble Space Telescope}

The atmospheric structure mapping efforts with IUE have been limited to the $\mathrm{Mg}$ II $\mathrm{h}$ and $\mathrm{k}$ emission lines, which are formed throughout the lower chromosphere. The ultraviolet spectrosopy capabilities of the Goddard High Resolution Spectrograph and the increased sensitivity of the Hubble Space Telescope can now being exploited to expand our studies to high-resolution observations of lines formed at several regions in the outer atmosphere.

AR Lac was observed with HST simultaneously with IUE, ROSAT, and the VLA in December 1991. Eight spectra of the C IV (1550 $\AA$ ) lines and the $\mathrm{Mg}$ II $\mathrm{h}$ and $\mathrm{k}$ lines were obtained with GHRS at uniform phase intervals throughout one of the cycles covered by IUE (Walter et al. 1993). The Mg II spectra contained no more information than the IUE data, even though they were of superior $\mathrm{S} / \mathrm{N}$. The C IV spectra were of fairly low $\mathrm{S} / \mathrm{N}$, and the resolution was inadequate for detailed imaging, but they present us with our first opportunity to study the spatial structure of the transition region.

In September 1993 we obtained GHRS spectra of the C IV and Mg II lines of V711 Tau, again in conjunction with a multiwavelength campaign including IUE data (Dempsey et al. 1996). In addition, we obtained spectra of the density-sensitive lines of Si III] $(1892 \AA)$ and C III] $(1908 \AA)$. Our plan was to generate maps of the chromosphere and transition region brightness as well as a map of the density structure. Unfortunately, our planned phase coverage was cut in half, and the resultant $\mathrm{S} / \mathrm{N}$ was far lower than anticipated. Nevertheless, interesting new results are leading us to reinterpret the existing IUE data (see Dempsey et al. in this volume).

The geosynchronous orbit and real-time operations mode of IUE have been ideal for arranging these very successful observing campaigns. Never- 
theless, its limited sensitivity, especially in the far ultraviolet where there are a multitude of lines from all atmospheric levels and virtually no continuum, permits only the lower chromosphere to be studied. With these two initial GHRS data sets, we have demonstrated the potential of HST for extending these studies to other atospheric levels. In early May 1996, the active star V824 Ara will be observed with HST continuously for 26 HST orbits while the star is in HST's "continuous viewing zone". If successful, we will for the first time derive maps of the chromosphere and transition region simultaneously. We will also attempt to image the coronal structure using the Fe XXI line at $1354 \AA$.

\section{References}

Busa I., Pagano I., Rodonò M., Neff J.E., 1995, in Cool Stars, Stellar Systems, and the Sun, ed. R. Pallavicini (ASP Conf. Series), in press

Collier Cameron A., 1992, in Surface Inhomogeneitites on Late-Type Stars, eds. P.B. Byrne \& D.J. Mullan (Springer: Berlin), p. 33

Dempsey R.C., Neff J.E., Linsky J.L., Brown A., 1996, ApJ, submitted

Linsky J.L., Neff J.E., Brown A., Gross B.D., Simon T., Andrews A.D., Rodonò M., Feldman P.A., 1989, A\&A, 211, 173

Neff J.E., 1990, in Active Close Binaries, ed. C. Ibanoğlu (Kluwer: Dordrecht), p. 805

Neff J.E., 1991, in The Sun and Cool Stars: Activity, Magnetism, and Dynamos, ed. I. Tuominen, (Springer-Verlag, Lecture Notes in Physics), p. 330

Neff J.E., 1992, in Surface Inhomogeneitites on Late-Type Stars, eds. P.B. Byrne \& D.J. Mullan (Springer: Berlin), p. 54

Neff J.E., Pagano I., Rodonò M., 1994, in Cool Stars, Stellar Systems, and the Sun, ed. J.-P. Caillault (ASP Conf. Series), p. 447

Neff J.E., Pagano I., Rodonò M., 1995, in 4th Workshop on Multi-Site Continuous Spectroscopy, eds. L. Huang et al. (Beijing Astronomical Observatory), p. 157

Neff J.E., Pagano I., Rodonò M., Fox D.C., Brown A., Dempsey R.C., Linsky J.L., 1995, $\mathrm{A} \& \mathrm{~A}$, in press

Neff J.E., Walter F.M., Rodonò M., Linsky J.L., 1989, A\&A, 215, 79

Pagano I., Rodonò M., Neff J.E., 1992, in Surface Inhomogeneitites on Late-Type Stars, eds. P.B. Byrne \& D.J. Mullan (Springer: Berlin), p. 315

Pagano I., Rodonò M., Neff J.E., 1994, in Cool Stars, Stellar Systems, and the Sun, ed. J.-P. Caillault (ASP Conf. Series), p. 450

Piskunov N.E., Tuominen I., Vilhu O., 1990, A\&A, 230, 363

Unruh Y.C., Collier Cameron A., 1995, MNRAS, 273, 1

Vogt S.S., Penrod G.D., Hatzes A.P., 1987, ApJ, 321, 496

Walter F.M., Neff J.E., Gibson D.M., Linsky J.L., Rodonò M., Gary D.E., Butler C.J., 1987, A\&A, 186, 241

Walter F.M., Neff J.E., Pagano I., Rodonò M., 1993, in UV and X-Ray Spectroscopy of Laboratory and Astrophysical Plasmas, eds. E. Silver \& S. Kahn (Cambridge Univ. Press), p. 403 